\title{
Doped graphene as tunable electron-phonon coupling material
}

\author{
Claudio Attaccalite ${ }^{1,2}$, Ludger Wirtz ${ }^{3}$, Michele \\ Lazzeri $^{4}$, Francesco Mauri ${ }^{4}$ and Angel Rubio ${ }^{1,5}$ \\ 1 Nano-Bio Spectroscopy group and ETSF Scientific Development Centre, \\ Dpto. Fisica de Materiales, Universidad del Pais Vasco, \\ Centro de Fisica de Materiales CSIC-UPV/EHU- \\ MPC and DIP, E-20018 San Sebastian, Spain \\ 2 Institut Neel, CNRS-UJF, Grenoble, France \\ 3 Institute for Electronics, Microelectronics, \\ and Nanotechnology, CNRS-UMR 8520, Dept. ISEN, \\ B.P. 60069, 59652 Villeneuve d'Ascq Cedex, France \\ 4 IMPMC, Universités Paris 6 et \%, CNRS, \\ IPGP, 140 rue de Lourmel, 75015 Paris, France \\ ${ }^{5}$ Fritz-Haber-Institut der Max-Planck-Gesellschaft, Berlin, Germany
}

(Dated: March 26, 2010)

\begin{abstract}
We present a new way to tune the electron-phonon coupling (EPC) in graphene by changing the deformation potential with electron/hole doping. We show the EPC for highest optical branch at the high symmetry point $\mathrm{K}$, acquires a strong dependency on the doping level due to electronelectron correlation not accounted in mean-field approaches. Such a dependency influences the dispersion (with respect to the laser energy) of the Raman D and 2D lines and the splitting of the 2D peak in multi-layer graphene. Finally this doping dependence opens the possibility to construct tunable electronic devices through the external control of the EPC.
\end{abstract}

PACS numbers: 71.15.Mb, 63.20.Kr, 78.30.Na, 81.05.Uw 
A large amount of work envisioning exciting new applications of graphene-based devices in nanoelectronics has been published in the last years (see Ref. 1 and references therein). The performance of those electro-optical graphene-based-devices ${ }^{2}$ is governed to a large extent by the electron-phonon coupling (EPC), or, more precisely, by the deformation potential. For example in high-current transport the scattering with phonons increases the differential resistance in carbon nanotubes and graphene $\mathrm{e}^{\underline{3}-\underline{5}}$. It has been widely assumed that the deformation potential in graphene is constant with respect to the electron/hole concentration. For a proper description of device performances it is necessary to control the validity of this approximation. Indeed, here we show that the deformation potential displays a rather strong doping dependence which should be taken into account in the design of new graphene devices.

The interaction between electrons and phonons in graphene and graphite has been studied with many experimental techniques ranging from Angle Resolved Photoemission Spectroscopy (ARPES $)^{7}$, inelastic x-ray scattering(IXS) ${ }^{8}$, Scanning Tunneling Spectroscopy $(\mathrm{STS})^{9}$ to Raman spectroscopy ${ }^{\underline{10}}$. In particular, Raman spectroscopy is commonly employed in graphene characterization because it is sensitive to the number of layers ${ }^{11}$, 2 , the doping level ${ }^{13}-\underline{18}$ and the graphene edges $\underline{19}$. In order to interpret all above mentioned experiments, a complete knowledge of the electronic structure, the phonon dispersion and the electronphonon interaction is required. In graphene the electron-phonon coupling(EPC) between the $\pi$ and $\pi^{*}$ bands is responsible for the peculiar properties observed in the experiments $20,21,23$.

The dimensionless electron-phonon coupling for a mode $\nu$ at momentum $\mathbf{q}$ is given by:

$$
\begin{array}{r}
\lambda_{\mathbf{q} \nu}=\frac{2}{\hbar \omega_{\mathbf{q} \nu} N_{\sigma}\left(\epsilon_{f}\right)} \int_{B Z} \frac{d \mathbf{k}}{\Omega} \sum_{i, j}\left|g_{\mathbf{k} i,(\mathbf{k}+\mathbf{q}) j}^{\nu}\right|^{2} \times \\
\delta\left(\epsilon_{k}-\epsilon_{f}\right) \cdot \delta\left(\epsilon_{k+q}-\epsilon_{f}\right)
\end{array}
$$

where $\omega_{q v}$ is the phonon frequency, $N_{\sigma}\left(\epsilon_{f}\right)$ is the density of states per spin channel at the Fermi level, and $i$ and $j$ are band indices. The term

$$
g_{\mathbf{k} i,(\mathbf{k}+\mathbf{q}) j}^{\nu}=\left\langle\mathbf{k}+\mathbf{q}, j\left|\Delta V_{\mathbf{q} \nu}\right| \mathbf{k}, i\right\rangle \sqrt{\hbar /\left(2 M \omega_{q}\right)}
$$

is the electron-phonon coupling matrix element that describes the scattering of an electron from band $i$ to band $j$ due to the phonon $\nu$ with wavevector $\mathbf{q}$. The quantity $\lambda_{\mathbf{q} \nu}$ depends on the doping of the system through the shift of the Fermi level and the subsequent change in $N_{\sigma}\left(\epsilon_{f}\right)$. Furthermore, it (weakly) depends on the doping through the variation of $g_{\mathbf{k} i,(\mathbf{k}+\mathbf{q}) j}^{\nu}$. 
In the following we are interested in the contribution coming from the matrix elements, therefore, in order to lift the dependence on the phonon frequency, we calculate directly $\left\langle\mathbf{k}+\mathbf{q}, j\left|\Delta V_{\mathbf{q} \nu}\right| \mathbf{k}, i\right\rangle$. In particular, we will concentrate on the coupling of the $\pi$ and $\pi^{*}$ bands with the highest optical phonon branch $(\mathrm{HOB})$ at $\boldsymbol{\Gamma}\left(\mathrm{E}_{2 g}\right.$ mode) and at $\mathbf{K}\left(\mathrm{A}_{1}^{\prime}\right.$ mode). We define

$$
\left\langle D_{\boldsymbol{\Gamma}}^{2}\right\rangle=\sum_{i, j}^{\pi, \pi^{*}}\left|\left\langle\mathbf{K}, j\left|\Delta V_{\boldsymbol{\Gamma} E_{2 g}}\right| \mathbf{K}, i\right\rangle\right|^{2} / 4
$$

and

$$
\left\langle D_{\mathbf{K}}^{2}\right\rangle_{\mathbf{k}}^{\pi \pi^{*}}=\left|\left\langle\mathbf{2} \mathbf{K}+\mathbf{k}, \pi^{*}\left|\Delta V_{\mathbf{K} A_{1}^{\prime}}\right| \mathbf{K}+\mathbf{k}, \pi\right\rangle\right|^{2} / 2
$$

where the sums are performed over the two times degenerate $\pi$ bands at $\mathbf{K}$. In the limit of zero doping, $\left\langle D_{\boldsymbol{\Gamma}}^{2}\right\rangle$ is equal to $\left\langle D_{\boldsymbol{\Gamma}}^{2}\right\rangle_{F}$ as defined in Ref. 27. In the limit of zero doping and $\mathbf{k} \rightarrow \mathbf{0},\left\langle D_{\mathbf{K}}^{2}\right\rangle_{\mathbf{k}}^{\pi \pi^{*}}$ is equal to $\left\langle D_{\mathbf{K}}^{2}\right\rangle_{F}$ as defined in Ref. 27. In fact, for small $\mathbf{k}$ the matrix elements between $\pi$ and $\pi$ (or between $\pi^{*}$ and $\pi^{*}$ ) are zero (see note [24] of Ref. 23). In our earlier publications ${ }^{27}$ we called these quantities electron-phonon coupling. In view of the above definitions (Eqs. (11) and (2) ) it is more precise to call $\left\langle D_{\mathbf{\Gamma}}^{2}\right\rangle$ and $\left\langle D_{\mathbf{K}}^{2}\right\rangle_{\mathbf{k}}^{\pi \pi^{*}}$ the "square of the deformation potential" (or, to be very precise, the "average squared deformation potential of the $\pi$-bands"). We focus our attention on $\left\langle D_{\mathbf{\Gamma}}^{2}\right\rangle$ and $\left\langle D_{\mathbf{K}}^{2}\right\rangle_{\mathbf{k}}^{\pi \pi^{*}}$, because these two quantities are the ones responsible for the intensity and position of the peaks in Raman spectroscopy ${ }^{10}$, the kinks in ARPES and the phonon slope close to the Kohn-anomalies ${ }^{23}$.

In graphene and carbon nanotubes, the deformation potential has been usually obtained from tight-binding Hamiltonians computing the change in the nearest-neighbor hopping energy due to a lattice distortion ${ }^{24}$. In this approach the deformation potential, has been always considered a constant with respect to the electron or hole density. This approximation, although not justified microscopically, is widely used $1,13,14,16,18,20,24,26$. Moreover even $a b$-initio calculations using Density Functional Theory (DFT) in the local density approximation (LDA) apparently confirmed that the deformation potential is weakly dependent on doping. However, including effects of electron-electron correlation, we will show that for the $\mathbf{K}$ ( $\mathrm{A}_{1}^{\prime}$ mode) mode, this approximation breaks down and $\left\langle D_{\mathbf{K}}^{2}\right\rangle_{\mathbf{k}}^{\pi \pi^{*}}$ can change by more than $40 \%$ just varying the electronic distribution (i.e. gated single and multi-layer graphene). This fact can be directly probed measuring the Raman D-peak dispersion of graphene versus doping as we will discuss in the following.

Recently it has been proven ${ }^{27,28}$ that in (neutral) graphene $\left\langle D_{\mathbf{K}}^{2}\right\rangle_{\mathbf{k}=\mathbf{0}}^{\pi \pi^{*}}$ is strongly affected 
by electron correlation. In our previous work ${ }^{27}$, we have shown that DFT-LDA or DFTGGA underestimates $\left\langle D_{\mathbf{K}}^{2}\right\rangle_{\mathbf{k}=\mathbf{0}}^{\pi \pi^{*}}$ by almost a factor of two. The electron-electron correlation can be included on the level of the GW-approximation obtaining a deformation potential which reproduces the Raman D-line dispersion and the phonon slope around $\mathbf{K}$ within few percent ${ }^{8}$. On the contrary, the deformation potential of the $\boldsymbol{\Gamma}-\mathrm{E}_{2 g}$ mode was shown to depend very little on electron-electron correlation ${ }^{27}$. In this letter, we use the GW-approximation to calculate the variation of the deformation potential with doping.

Doped graphene can be created in single layer field-effect transistor (FET) based experiments where an electron concentration up to $3 \cdot 10^{13} \mathrm{~cm}^{-2}$ electron can be realized, while higher dopings are obtained using intercaled graphite ${ }^{29}$. In order to simulate doped graphene we employed a slab-geometry, i.e., bulk geometry with large distance between the layers, changing the number of electrons in the unit cell and then compensating the negative/positive charge with a uniform positive/negative background, see Ref. 30 for details. The electronic and phonon structure of graphene at different doping levels were computed using DFT-LDA. The deformation potential is obtained using the scheme proposed in Ref. 27 based on a frozen-phonon approach by looking at the modification of the electronic structure upon displacement of the atoms following a given normal mode. The major advantage of this approach is that it can be used with electronic structure methods other than DFT. When DFT is used, this approach gives the same result of density functional perturbation theory(DFPT). First of all, we investigated the effect of the change in the lattice constant $a$, induced by the doping, on the deformation potential. Using the functional dependence of $a$ versus the electron concentration from Eq. (2) of Ref. 31, we calculated the deformation potential for different doping level with the corresponding lattice parameters. In panel b of Fig. 1 we compare the deformation potential calculated with and without lattice relaxation for different electron/hole doping. The difference between the two results is small when compared with renormalization effects that we are going to describe below. Therefore, in order to make the analysis simpler, we performed all the calculations with the graphene experimental lattice constant.

On the level of the LDA, the variation of $\left\langle D_{\mathbf{K}}^{2}\right\rangle_{\mathbf{k}}^{\pi \pi^{*}}$ and $\left\langle D_{\boldsymbol{\Gamma}}^{2}\right\rangle$ with doping is very small. In the following, we will introduce correlation effects beyond DFT-LDA on the deformation potential. We included these effects in $G W$ approximation ${ }^{34}$ that has been successfully applied in the study of graphene and graphite ${ }^{32,33}$ and its compounds ${ }^{36,37}$. First of all we 


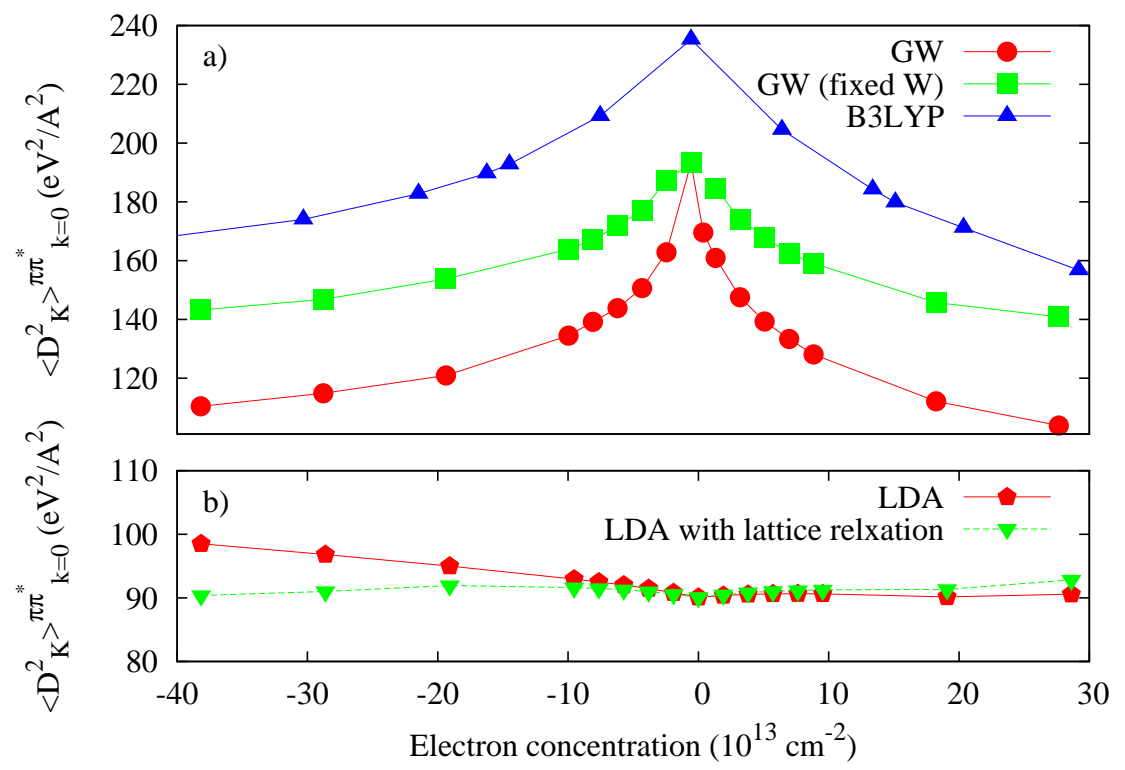

FIG. 1: Panel $a$ : squared deformation potential for the $\mathbf{K}-\mathrm{A}_{1}^{\prime}$ phonon between the $\pi$ bands $\left\langle D_{\mathbf{K}}^{2}\right\rangle_{\mathbf{k}=\mathbf{0}}^{\pi \pi^{*}}$ in different approximations. Circles are $G W$ results; squares are $G W(0)$ ones, keeping the screened potential $W(0)$ fixed to the undoped case; triangles are B3LYP results. Panel $b$ : variation of $\left\langle D_{\mathbf{K}}^{2}\right\rangle_{\mathbf{k}=\mathbf{0}}^{\pi \pi^{*}}$ in LDA approximation, including or not lattice relaxation (triangles and pentagons, respectively).

studied the quasiparticle band-structure versus doping (see also Refs. 41 and 42 ), that will be subsequently used to calculate the D-peak dispersion. In table $\mathbb{1}$, we report the change in the gap between the $\pi$ and $\pi^{*}$ at the high symmetry point $\mathbf{M}, \Delta \epsilon_{M}$, as function of doping. This quantity is directly related to the optical properties of graphite and graphene-based materials, and provides an alternative way to measure quasi-particle renormalization effects. The strongest renormalization effect (compared to the LDA-gap) is present for zero doping. Electron/hole doping rapidly decreases the GW renormalization of the quasi-particle band structure. The same is true for the Fermi velocity $v_{F}$. Then from the change in the quasiparticle band structure upon atomic displacement, we calculated $\left\langle D_{\mathbf{K}}^{2}\right\rangle_{\mathbf{k}}^{\pi \pi^{*}}$ in the same way as was done for LDA (see ref. 27). We found that for $\left\langle D_{\Gamma}^{2}\right\rangle$ the GW result of ref.27 is mainly unaffected by the doping level ${ }^{38}$. The situation is completely different for $\left\langle D_{\mathbf{K}}^{2}\right\rangle_{\mathbf{k}}^{\pi \pi^{*}}$. In Fig. 1, and table I, we report the value of $\left\langle D_{\mathbf{K}}^{2}\right\rangle_{\mathbf{k}=\mathbf{0}}^{\pi \pi^{*}}$ as a function of the doping in different approximations. In LDA (panel b) it is almost a constant, but the situation is completely different at the GW level (panel a): $\left\langle D_{\mathbf{K}}^{2}\right\rangle_{\mathbf{k}=\mathbf{0}}^{\pi \pi^{*}}$ is increased more than $80 \%$ with respect to the 
TABLE I: Squared deformation potential for the $\mathbf{K}-\mathrm{A}_{1}^{\prime}$ phonon versus doping, in $G W$ approximation. $\left\langle D_{\mathbf{K}}^{2}\right\rangle_{\mathbf{k}=\mathbf{0}}^{\pi \pi^{*}}$ and $\Delta \epsilon_{\mathbf{M}}$ are defined in the text, the LDA value of $\Delta \epsilon_{\mathbf{M}}$ at zero doping is $4.01115 \mathrm{eV}$. D-slope is the slope of the Raman D-peak dispersion (peak energy versus laser frequency), see the text.

\begin{tabular}{|c|c|c|c|}
\hline \multirow{3}{*}{$\begin{array}{c}\Delta n \\
10^{13} \mathrm{~cm}^{-2}\end{array}$} & \multicolumn{3}{|c|}{ Graphene: } \\
\hline & $\left\langle D_{\mathbf{K}}^{2}\right\rangle_{\mathbf{k}=\mathbf{0}}^{\pi \pi^{*}}$ & $\Delta \epsilon_{\mathbf{M}}$ & D-slope \\
\hline & $\left(\mathrm{eV}^{2} / \AA^{2}\right)$ & $\mathrm{eV}$ & $\mathrm{cm}^{-1} / \mathrm{eV}$ \\
\hline-38.16 & 110 & 4.550 & 27.58 \\
\hline-28.62 & 115 & 4.585 & 29.83 \\
\hline-19.08 & 121 & 4.632 & 32.41 \\
\hline-9.54 & 135 & 4.711 & 37.84 \\
\hline-7.63 & 139 & 4.723 & 39.49 \\
\hline-5.72 & 144 & 4.761 & 41.20 \\
\hline-3.81 & 151 & 4.795 & 43.67 \\
\hline-1.90 & 163 & 4.820 & 48.20 \\
\hline 0.00 & 193 & 4.867 & 58.40 \\
\hline 1.90 & 161 & 4.803 & 47.82 \\
\hline 3.81 & 148 & 4.765 & 43.26 \\
\hline 5.72 & 139 & 4.724 & 40.24 \\
\hline 7.63 & 133 & 4.667 & 38.44 \\
\hline 9.54 & 128 & 4.642 & 36.75 \\
\hline 19.08 & 112 & 4.532 & 31.53 \\
\hline 28.62 & 104 & 4.485 & 29.11 \\
\hline
\end{tabular}

LDA result (at zero doping) and it acquires a strong doping dependence. The renormalized deformation potential rapidly decreases with electron or hole doping and gets close to the LDA value at large doping.

In the following, we discuss the origin of the strong doping dependence of $\left\langle D_{\mathbf{K}}^{2}\right\rangle_{\mathbf{k}=\mathbf{0}}^{\pi \pi^{*}}$ within the GW-approximation. Both the Green's function, $G$, and the screened Coulomb potential, $W$, are doping dependent. In order to disentangle the two effects, we performed test calculations within the GW approximation keeping the screened Coulomb interaction fixed 
to its value at zero doping. The result is shown in Fig. 1. The doping dependence of the deformation potential is reduced by about a factor of two. Therefore, we conclude that the role of the screening is comparable to the correction coming from the Green's function variation. In fact the shift of the Fermi level due to doping, leads to a suppression of transitions from $\pi$ to $\pi^{*}$ states. This affects the deformation potential both through a change of the screening and of the Green's function.

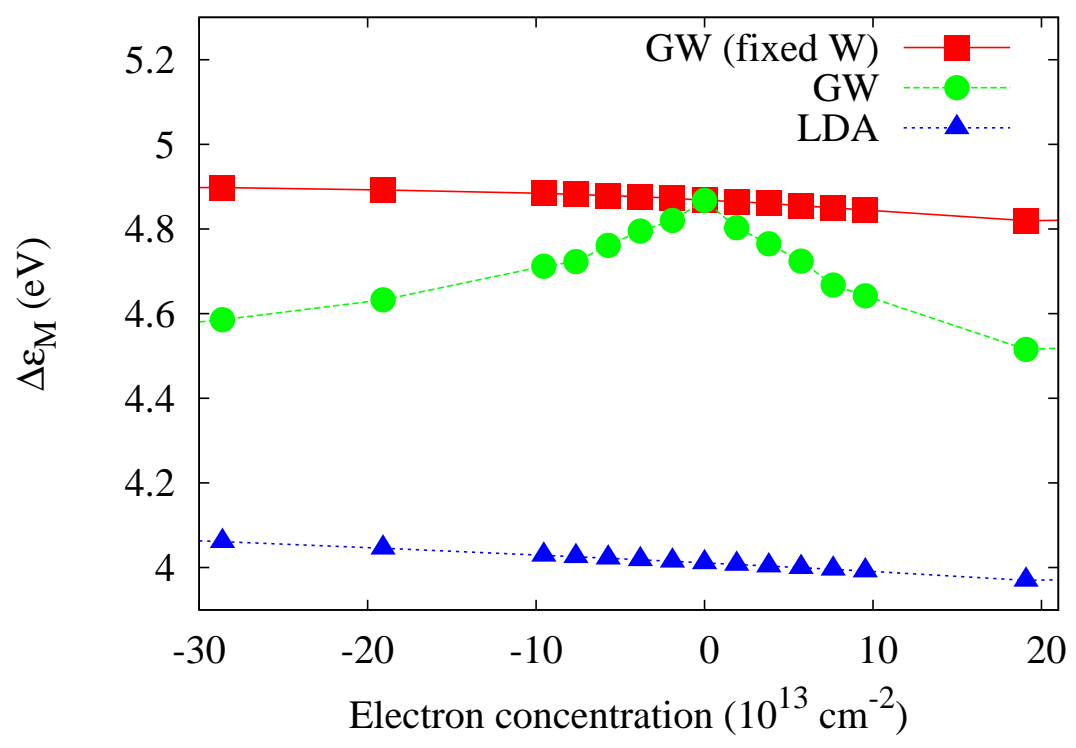

FIG. 2: Electronic gap at $M$ point $\left(\Delta \epsilon_{\mathbf{M}}\right)$ as function of doping in different approximations: GW with fixed screened Coulomb interaction, GW, and LDA

We compare the result for deformation potential with the one of the $\Delta \epsilon_{M}$ gap. For the latter we found that all the doping dependence is due to the screened Coulomb potential and not due to the change in the Green's function, see fig. 2, while in the first both effects contribute to the its behavior with doping. This is due to the fact that, for the $\mathbf{K}-\mathrm{A}_{1}^{\prime}$ phonon, GW not only renormalizes the bare Green function lines but also introduces vertex corrections that behave different with the doping. However in general this is not true for other phonon modes, for instance for the $\boldsymbol{\Gamma}-\mathrm{E}_{2 g}$ mode vertex corrections have been proven to be negligible $e^{28}$.

At this point it is instructive to check the performance of DFT with hybrid functionals. We have performed calculations with the B3LYP functiona $2^{39}, 40$. Apart from an overestimation of $\left\langle D_{\mathbf{K}}^{2}\right\rangle_{\mathbf{k}=0}^{\pi \pi^{*}}$ by about $25 \%$ (which could be corrected for by diminishing the percentage 
of Hartree-Fock exchange in the functional), the calculation reflects rather the doping dependence of the GW-calculation with constant screening than the dependence of the full GW-calculation. This can be understood, because the B3LYP functional contains screening on a very simplified level not suitable to describe extended systems and metals.

Now we want show how the variation of the deformation potential affects the Raman D peak dispersion and the splitting of the 2D line in multilayer graphene. The dispersion (peak energy versus laser frequency) of the Raman D and 2D lines in graphene is conveniently described by the double-resonant Raman model ${ }^{21}$. In order to calculate the phonon dispersion we need the deformation potential for a phonon wavevector $\left.\mathbf{K}+\mathbf{q}\left(\left\langle D_{\mathbf{K}+\mathbf{q}}^{2}\right\rangle\right\rangle_{\mathbf{k}}^{\pi \pi^{*}}\right)$. In our earlier works we assumed this as a constant in $\mathbf{q}$ and $\mathbf{k}$. The calculation for a finite $\mathbf{q}$ requires the use of very large supercells, which are challenging for the GW approach. On the contrary, we can test the dependence of the deformation potential on the electron wavevector $\mathbf{k}$. Indeed, we calculated $\left\langle D_{\mathbf{K}}^{2}\right\rangle_{\mathbf{k}}^{\pi \pi^{*}}$ for $\mathbf{k}$ varying along the line $\boldsymbol{\Gamma}-\mathbf{M}^{22}$. We found that $\left\langle D_{\mathbf{K}}^{2}\right\rangle_{\mathbf{k}}^{\pi \pi^{*}}$ is almost constant in the doped case and it is slightly varying in the undoped one (within 10\%), the result is reported in fig. 3. This finding justifies our previous assumption 27 of a constant square deformation potential that was also verified by direct comparison with the experiments ${ }^{8}$. In the double-resonant Raman model ${ }^{21}$ the D-line dispersion is proportional to the phonon slope around $\mathbf{K}$ and thus proportional to $\left\langle D_{\mathbf{K}}^{2}\right\rangle_{\mathbf{k}}^{\pi \pi^{*}}$. Furthermore, it is inversely proportional to the slope of the $\pi / \pi^{*}$ bands and thus inversely proportional to $\Delta \epsilon_{m}$. In our previous work using the result at zero doping, we were able to reproduce completely ab-initio the Raman D-peak ${ }^{27}$ dispersion (peak position versus laser frequency). With the information on the doping dependence of $\left\langle D_{\mathbf{K}}^{2}\right\rangle_{\mathbf{k}}^{\pi \pi^{*}}$ and of $\Delta \epsilon_{m}$, we have calculated the Raman D-peak dispersion as a function of the doping with the approach described in ref. 27. In Fig. 4 we report the resulting slope of the D-peak dispersion obtained as a linear fit of the dispersion between 1.0 and $3.2 \mathrm{eV}$ laser energy. The D-peak dispersion is almost symmetric with respect to electron/hole doping, and it has its maximum at zero doping. Due to this strong variation with doping it can be used also to detect experimentally the charge state of a graphene sample. Finally we suggest a simple way to verify our result in multi-layer graphene using a single laser $\underline{\underline{43}}$. In multilayer graphene the $2 \mathrm{D}$ peak splits in different sub-peaks and this splitting is proportional to the D-peak dispersion with the laser frequency $\underline{\underline{11}}, \underline{12}$. Therefore we expect that measurements of the 2D peak splitting as function of doping can highlight the strong variation of the D-peak dispersion, due to the squared 


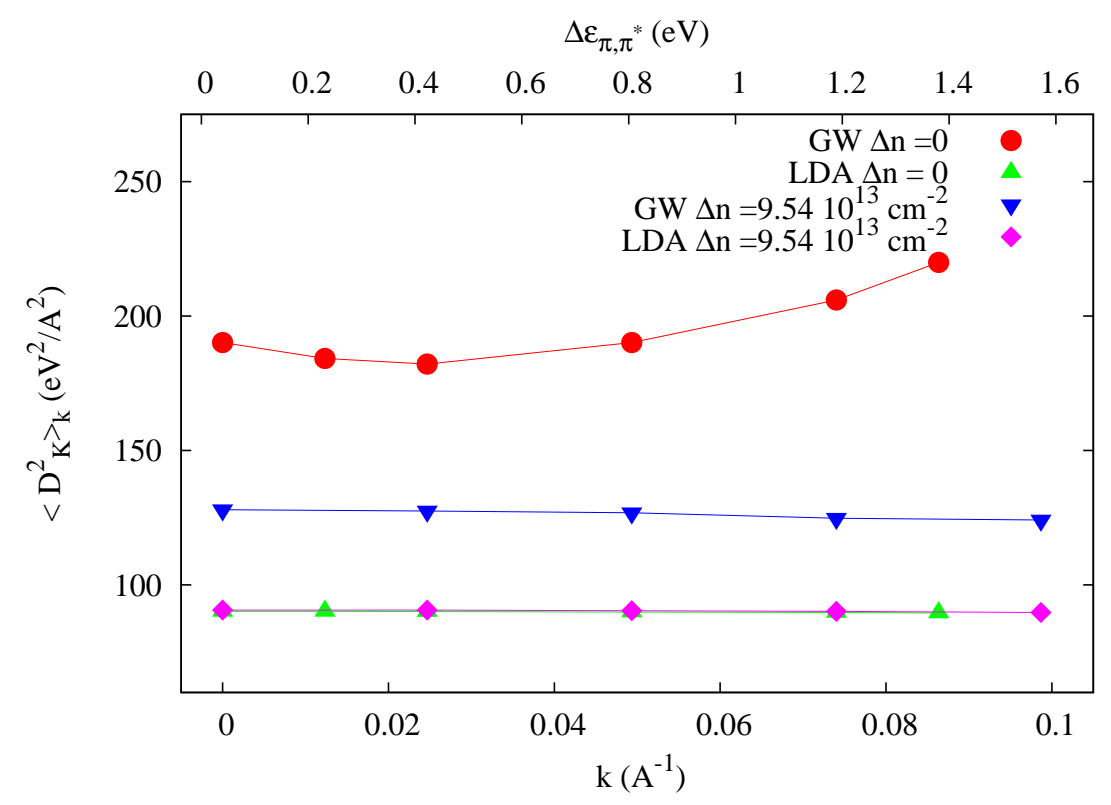

FIG. 3: Square of the deformation potential, $\left\langle D_{\mathbf{K}}^{2}\right\rangle_{\mathbf{k}}^{\pi \pi^{*}}$, with $\mathbf{k}$ varying along the line $\mathbf{\Gamma}-\mathbf{M}$, perpendicular to $\boldsymbol{\Gamma}-\mathbf{K}$. Four cases are reported: circles and up triangles are GW and LDA results for the undoped case; down-triangles and rhombus are GW and LDA for high doping $\Delta n=9.5410^{13} \mathrm{~cm}^{-2}$. The upper labels refere to the corresponding electronic gap (the energy difference between $\pi^{*}$ and $\pi$ bands at $\mathbf{K}+\mathbf{k}$ according to GW calculations at zero doping).

deformation potential, as predicted in this work.

In conclusion, we have shown that in graphene $\left\langle D_{\mathbf{K}}^{2}\right\rangle_{\mathbf{k}}^{\pi \pi^{*}}$ can be easily tuned with doping. This means that the electron and phonon interaction cannot be described by a simplified Hamiltonian with a fixed deformation potential as it is commonly done. Our findings can be easily verified experimentally by measuring the doping dependence of the Raman Dpeak dispersion, of the splitting of the $2 \mathrm{D}$ peak in multi-layer graphene and of the slope of the highest phonon branch close to $\mathbf{K}$. The present findings open the possibility to use the slope of the D-peak dispersion as a simple probe for the electrons/holes doping in graphene. The fact that the deformation potential is not a constant has implications also in the realization of graphene-based electronic devices where it is known that one of the limitation for ballistic transport is just the coupling between electrons and phonons $\mathrm{s}^{\frac{3}{-5}}$. Tuning electron-phonon coupling by doping could boost electronic properties of graphene based devices. Concerning the puzzling discussion on the size of the EPC in graphene, our result puts another piece in support that EPC has to be larger than the LDA one and doping 


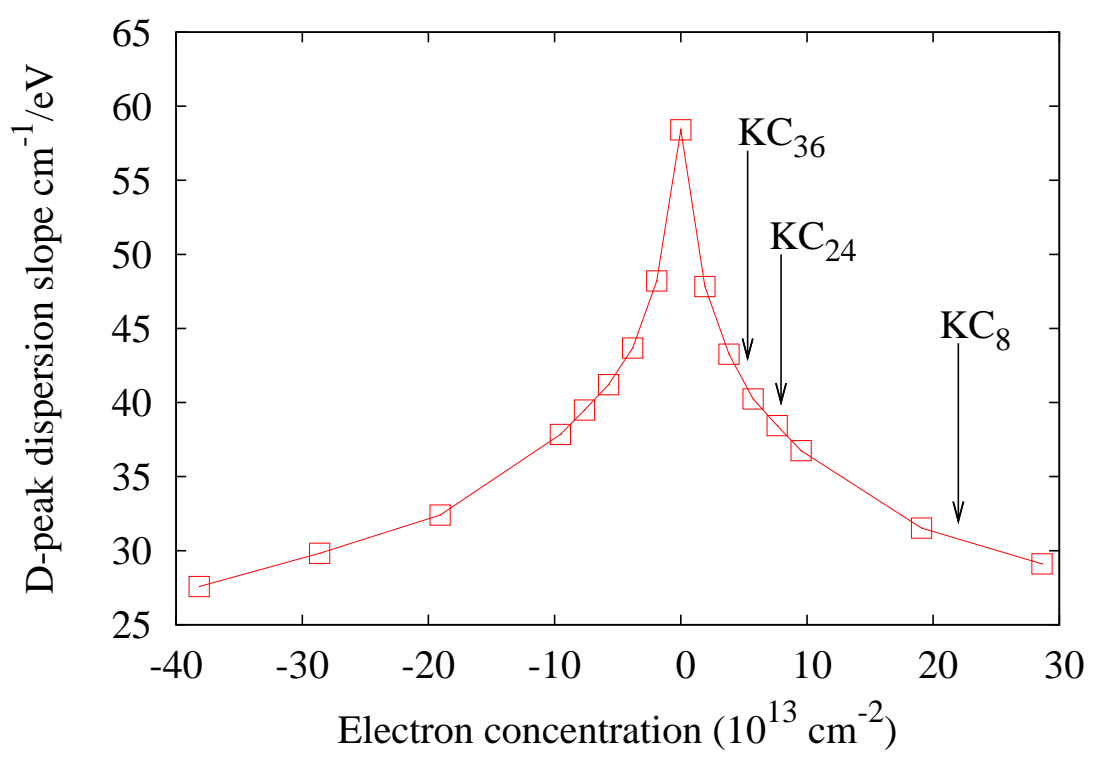

FIG. 4: Change of slope of the Raman D-peak dispersion versus doping. Arrows indicate the equivalent doping level for the $\mathrm{KC}_{8}, \mathrm{KC}_{24}, \mathrm{KC}_{36}$ intercalated graphite.

dependent. Finally, this new way to tune electron-phonon coupling can play a fundamental role in all the experiments where physical phenomena are directly related to doping as for instance the phonon renormalization $\underline{6}$, phonon linewitdh $\stackrel{45}{ }$, or the radiative decay of excitons in nanotubes $\underline{46}$.

We acknowledge funding by the Spanish MEC (FIS2007-65702-C02-01), "Grupos Consolidados UPV/EHU del Gobierno Vasco" (IT-319-07), the European Community through e-I3 ETSF project (Contract Number 211956), the french ANR project ACCATTONE. We acknowledge support by the Barcelona Supercomputing Center, "Red Espanola de Supercomputacion", SGIker ARINA (UPV/EHU) and Transnational Access Programme HPCEurope++.

1 A. H. Castro Neto, F. Guinea,N. M. R. Peres,K. S. Novoselov and A. K. Geim, Rev. Mod. Phys. 81, 109 (2009)

2 Tsang, et al., Nature nanotechnology 2, 725 (2007)

3 Z. Yao et al., Phys. Rev. Lett. 84, 2941 (2000); A. Javey et al., Phys. Rev. Lett. 92, 106804 (2004) 
4 M. Lazzeri, S. Piscanec, F. Mauri, A. C. Ferrari, and J. Robertson, Phys. Rev. Lett. 95, 236802 (2005), B.J. LeRoy, S.G. Lemay, J. Kong, and C. Dekker, Nature (London) 432, 371 (2004)

5 A. Barreiro, M. Lazzeri, J. Moser, F. Mauri, and A. Bachtold, Phys. Rev. Lett. 103, 076601 (2009)

6 A. B. Kuzmenko, L. Benfatto, E. Cappelluti, I. Crassee, D. van der Marel, P. Blake, K. S. Novoselov, Phys. Rev. Lett. 103, 116804 (2009)

7 A. Grüneis, C. Attaccalite, A. Rubio, D. V. Vyalikh, S. L. Molodtsov, J. Fink, R. Follath, W. Eberhardt, B. Büchner, and T. Pichler, Phys. Rev. B 79, 205106 (2009)

8 A. Grüneis, J. Serrano, A. Bosak, M. Lazzeri, S. L. Molodtsov, L. Wirtz, C. Attaccalite, M. Krisch, A. Rubio, F. Mauri, and T. Pichler, Phys. Rev. B 80, 085423 (2009)

9 Guohong Li, Adina Luican and Eva Y. Andrei, Phys. Rev. Lett. 102, 176804 (2009)

10 A. Das, et al., Nature Nano 3, 210 (2008), A. Das, Phys. Rev. B 79, 155417 (2009)

11 A.C. Ferrari, J.C. Meyer, V. Scardaci, C. Casiraghi, M. Lazzeri, F. Mauri, S. Piscanec, D. Jiang, K. S. Novoselov, S. Roth, and A. K. Geim, Phys. Rev. Lett. 97, 187401 (2006)

12 D. Graf, F. Molitor, K. Ensslin, C. Stampfer, A. Jungen, C. Hierold, and L. Wirtz, Nano Lett. 7, $238(2007)$

13 S. Pisana, M. Lazzeri, C. Casiraghi, K. S. Novoselov, A. K. Geim, A. C. Ferrari, and F. Mauri, Nature Mat. 6, 198 (2007).

14 J. Yan, Y. Zhang, P. Kim, and A. Pinczuk, Phys. Rev. Lett. 98, 166802 (2007)

15 C. Casiraghi, S. Pisana, K.S. Novoselov, A.K. Geim, and A.C. Ferrari, Appl. Phys. Lett. 91, 233108 (2007).

16 C. Stampfer, F. Molitor, D. Graf, K. Ensslin, A. Jungen, C. Hierold, and L. Wirtz, Appl. Phys. Lett. 91, 241907 (2007)

17 D. M. Basko, S. Piscanec, A. C. Ferrari, Phys. Rev. B 80, 165413 (2009)

18 M. Lazzeri and F. Mauri, Phys. Rev. Lett. 97, 266407 (2006)

19 C. Casiraghi, A. Hartschuh, H. Qian, S. Piscanec, C. Georgi, K. S. Novoselov, D. M. Basko, A. C. Ferrari, Nano Lett., 9, 1433 (2009)

20 Matteo Calandra and Francesco Mauri, Phys. Rev. B 76, 205411 (2007)

21 C. Thomsen and S. Reich, Phys. Rev. Lett. 85, 5214 (2000).

22 We consider $\mathbf{k}$ along the line $\boldsymbol{\Gamma}-\mathbf{M}$, perpendicular to $\boldsymbol{\Gamma}-\mathbf{K}$, of the graphene unit-cell. $\left\langle D_{\mathbf{K}}^{2}\right\rangle_{\mathbf{k}}^{\pi \pi^{*}}$ can be obtained from the band energies of a $\sqrt{3} \times \sqrt{3}$ supercell (see Fig $3 \mathrm{~b}$ of Ref ${ }^{27}$ ). The two 
Dirac cones at $\mathbf{K}$ and $2 \mathbf{K}$ of the unit-cell are refolded at $\boldsymbol{\Gamma}$ in the supercell. We dispalce each atom by $d$, following the $\mathbf{K}-\mathrm{A}^{\prime}{ }_{1}$ phonon pattern. We define $\Delta \epsilon_{d}(\mathbf{k})=\bar{\epsilon}_{\pi^{*}}(\mathbf{k})-\bar{\epsilon}_{\pi}(\mathbf{k})$, where $\bar{\epsilon}_{\pi^{*}}\left(\bar{\epsilon}_{\pi}\right)$ is the average between the energy of the two $\pi^{*}(\pi)$ bands corresponding to $\mathbf{K}+\mathbf{k}$ and $2 \mathbf{K}+\mathbf{k}$ of the unit-cell $\left\langle D_{\mathbf{K}}^{2}\right\rangle_{\mathbf{k}}^{\pi \pi^{*}}=\left(\left(\Delta \epsilon_{d}(\mathbf{k})\right)^{2}-\left(\Delta \epsilon_{0}(\mathbf{k})\right)^{2}\right) /\left(8 d^{2}\right)$.

23 S. Piscanec, M. Lazzeri, F. Mauri, A.C. Ferrari, and J. Robertson, Phys. Rev. Lett. 93, 185503 (2004).

24 K. Ishikawa and T. Ando, J. Phys. Soc. of Japan, 75, 084713 (2006)

25 S.K. Saha, U.V. Waghmare, H.R. Krishnamurthy, and A.K. Sood, Phys. Rev. B 76, 201404 (2007).

26 W. K. Tse and S. Das Sarma, Phys. Rev. Lett. 99, 236802 (2007)

27 M. Lazzeri, C. Attaccalite , L. Wirtz, and F. Mauri Phys. Rev. B 78, 081406(R) (2008)

28 D. M. Basko and I. L. Aleiner, Phys. Rev. B 77, 041409(R) (2008).

29 A. Grüneis, C. Attaccalite, A. Rubio, D. V. Vyalikh, S. L. Molodtsov, J. Fink, R. Follath, W. Eberhardt, B. Büchner, and T. Pichler, Phys. Rev. B 80, 075431 (2009)

30 In all DFT calculation the distance between the graphene planes was 20 a.u., the Brillouin Zone integration was performed using an uniform k-point grid $36 x 36 x 1$, with the functional of Ref. 47, plane waves (60 Ry cut-off) and pseudo-potentials $\stackrel{48}{ }$, using the PWSCF code $\underline{49}$. An electronic smearing of 0.02 Ry with the Fermi-Dirac distribution was employed.

31 M. Lazzeri, F. Mauri Phys. Rev. Lett. 97, 266407 (2006)

32 C. H. Park, F. Giustino, M. L. Cohen and S. G.. Louie, Phys. Rev. Lett. 99, 086804 (2007)

33 A. Grüneis et al., Phys. Rev. B, 78, 205425 (2008)

34 Non-self consistent GW calculations have been performed starting from DFT-LDA wavefunctions, using a plasmon pole approximation, following the scheme of Hybertsen and Louie ${ }^{35}$, with the code $\mathrm{YAMBO}^{50}$. We use a 36x36x1 k-point grid for the primitive cell and an equivalent one for the supercell. Convergence in the number of bands and size of the dielectric constant has been carefully checked.

35 M.S. Hybertsen and S.G. Louie, Phys. Rev. B 34, 5390 (1986).

36 S.Y. Zhou et al., Nature Phys. 2, 595-599 (2006)

37 A. Grüneis et al., Phys. Rev. Lett. 100, 037601 (2008)

38 Notice that non-adiabatic corrections, not considered here, are though to be important for the G phonon but not for the $\mathbf{K}$ phonon, see Ref. 31 
39 A.D. Becke, J. Chem. Phys. 98, 5648 (1993).

40 For the B3LYP calculations we used the code CRYSTAL (V.R. Saunders et al., R. Dovesi, C. Roetti, R. Orlando, C.M. Zicovich-Wilson, N.M. Harrison, K. Doll, B. Civalleri, I.J. Bush, Ph. DArco, M. Llunell CRYSTAL03 Users Manual, University of Torino, Torino, 2003), using the TZ basis by Dunning (without the diffuse P-function). K-point sampling and thermal smearing are the same as in the GW-calculations.

41 M. Polini, R. Asgari, Y. Barlas, T. Pereg-Barnea, A.H. MacDonald, Solid State Communications $143,58(2007)$

42 C. Attaccalite, A. Rubio, Physica Status Solidi B 246, 2523 (2009)

43 We suppose that also in multi-layer graphene, althougt the band structure it is different from graphene, a result similar to the one we found for graphen holds, as we found for graphite 27.

44 C. Faugeras, M. Amado, P. Kossacki, M. Orlita, M. Sprinkle, C. Berger, W.A. de Heer, M. Potemski, Phys. Rev. Lett. 103, 186803, (2009)

45 C. H. Park, F. Giustino, M. L. Cohen, S. G. Louie, Nano Lett. 8, 4229 (2008)

46 V. Perebeinos and P. Avouris, Phys. Rev. Lett. 101, 057401 (2008)

47 D.M. Ceperley and B.J. Alder, Phys. Rev. Lett. 45, 566 (1980)

48 N. Troullier and J. L. Martins, Phys. Rev. B 43, 1993 (1991)

49 P. Giannozzi et al. J. Phys.: Condens. Matter 21, 395502 (2009), http://www.quantum-espresso.org

50 A. Marini, C. Hogan, M. Gruning, and D. Varsano, Comp. Phys. Comm. 180, 1392 (2009), http://www.yambo-code.org 